\title{
Comment on 'The potential contribution of tumour-related factors to the development of FOLFOX-induced sinusoidal obstruction syndrome'
}

\section{Intraperitoneal FOLFOX does not consistently induce sinusoidal obstruction syndrome in C57BL/6 mice}

Claude Lentschener ${ }^{\star}, 1$, Carole Nicco ${ }^{2}$, Benoit Terris ${ }^{3}$, Charles-Marc Samama ${ }^{1}$ and Romain Coriat ${ }^{2,4}$

${ }^{1}$ Department of Anesthesia and Critical Care, Paris Descartes University, Assistance Publique-Hôpitaux de Paris, Cochin University Hospital, EA 3623 Paris, France; ${ }^{2}$ INSERM U1016 and CNRS UMR8104, Paris Descartes University, Assistance Publique-Hôpitaux de Paris, Cochin University Hospital, EA 3623 Paris, France; ${ }^{3}$ Department of Histopathology, Paris Descartes University, Assistance Publique-Hôpitaux de Paris, Cochin University Hospital, EA 3623 Paris, France and ${ }^{4}$ Department of Gastroenterology and Digestive Oncology, Paris Descartes University, Assistance Publique-Hôpitaux de Paris, Cochin University Hospital, EA 3623 Paris, France

Sir,

To our knowledge, Robinson et al (2013a, b) twice reported in 2013 the only animal model of sinusoidal obstruction syndrome (SOS) induced by FOLFOX (oxaliplatin, 5-fluoro-uracyl, folinic acid) administration. In this model, intraperitoneal FOLFOX administration had consistently induced SOS in 21 mice (Robinson et al, 2013a, b).

Prior to implementing a study in our institutional laboratory, we checked the reproducibility of Robinson et al's (2013a, b) procedures. Experiments were approved by the Veterinary Office of our university and carried out in accordance with institutional animal care guidelines. In line with Robinson et al (2013a), 10 C57BL/6 mice (Janvier Laboratory, Le Genest-Saint-Isle, France) between 8 and 10 weeks of age were housed in the animal facility of our hospital, had unrestricted access to standard diet (Global rodent diet-2016, Harlan Laboratories, Madison, WI, USA) and water, were maintained on a $12 \mathrm{~h}$ light/darkness cycle at a controlled room temperature $\left(21 \pm 2{ }^{\circ} \mathrm{C}\right)$ and humidity (between 40 and 60\%), and had an acclimation period of 1 week. Intraperitoneal oxaliplatin (Eloxatine, Sanofi-Aventis Laboratory, Gentilly, France) $6 \mathrm{mg} \mathrm{kg}^{-1}$, followed $2 \mathrm{~h}$ later by 5 -fluoro-uracyl (Fluorouracile Meram, Coopération Pharmaceutique Française, Melun, France) $50 \mathrm{mg} \mathrm{kg}^{-1}$, and folinic acid (Elvorine, Wyeth-Lederle, Catania, Italia) $90 \mathrm{mg} \mathrm{kg}^{-1}$, were prepared immediately before use and injected intraperitoneally in a volume of $250 \mathrm{ml}$, during the light cycle, on a weekly basis, for 5 weeks (Robinson et al, 2013a). One week after the final dose, hepatectomy was carried out under isoflurane inhalation anaesthesia.

One mouse died. Liver tissue was immersion-fixed in $4 \%$ buffered formaldehyde, embedded in paraffin, sectioned and stained with hematoxylin-eosin and Sirius red using standard histological techniques (Robinson et al, 2013a). Liver sections did not display any steatosis, inflammation, sinusoidal dilatation, perisinusoidal fibrosis, fibrotic venular occlusion or disrupted hepatocellular morphology that would be characteristic of any parenchyma alteration including SOS.

SOS is frequently observed on the healthy remnant parenchyma in patients undergoing liver resection for colorectal cancer metastasis as a side effect of FOLFOX chemotherapy (Soubrane et al, 2010). An animal model allowing SOS to be investigated independently of the effect of any confounding factors was deemed necessary. The present investigation of identical C57BL/6 mice, receiving an identical intraperitoneal chemotherapy regimen over an identical time duration, as reported by Robinson et al (2013a, b) to have consistently induced SOS, failed to induce SOS in any mice. Diet alters only steatotic changes in the liver and does not alter SOS features (Vetelainen et al, 2007). Only a diet, specifically deficient in one or more of the essential amino acids, limits monocrotaline-induced SOS in rats (Conotte and Colet, 2014). Robinson et al $(2013 \mathrm{a}, \mathrm{b})$ did not administer such a diet.

In contrast with previously reported (Robinson et al, 2013a, b), intraperitoneal FOLFOX does not consistently induce SOS in C57BL/6 mice. The discrepancy between our results and the results reported twice by Robinson et al (2013a, b) must be taken into account prior to implementing SOS investigation in a mouse model.

\section{ACKNOWLEDGEMENTS}

This study received Institutional funding.

\section{CONFLICT OF INTEREST}

The authors declare no conflict of interest.

\section{AUTHOR CONTRIBUTIONS}

All authors have contributed to the study design, the interpretation of results, and have critically revised and approved the text. CL and RC coordinated the study design and the investigation, and wrote the final manuscript. $\mathrm{RC}$ and $\mathrm{CN}$ carried out the FOLFOX intraperitoneal administration. BT did the histologic assessment. C-MS has helped in the organisation of the study, and has reviewed the final manuscript several times.

\section{REFERENCES}

Conotte R, Colet JM (2014) A metabonomic evaluation of the monocrotaline-induced sinusoidal obstruction syndrome (SOS) in rats. Toxicol Appl Pharmacol 276: 147-156.

Robinson SM, Mann DA, Manas DM, Oakley, Mann FJ, White SA (2013b) The potential contribution of tumour-related factors to the development of FOLFOX-induced sinusoidal obstruction syndrome. Br J Cancer 109: 2396-2403.

Robinson SM, Mann J, Vasilaki A, Mathers J, Burt AD, Oakley F, White SA, Mann DA (2013a) Pathogenesis of FOLFOX induced sinusoidal obstruction syndrome in a murine chemotherapy model. J Hepatol 59: 318-326.

Soubrane O, Brouquet A, Zalinski S, Terris B, Brezault C, Mallet V, Goldwasser F, Scatton O (2010) Predicting high grade lesions of sinusoidal obstruction syndrome related to oxaliplatin-based chemotherapy for colorectal liver metastases correlation with post-hepatectomy outcome. Ann Surg 251: 454-460.

Vetelainen R, Bennink RJ, van Vliet AK, van Gulik TM (2007) Mild steatosis impairs functional recovery after liver resection in an experimental model. Br J Surg 94: 1002-1008.

This work is published under the BJC's standard license to publish agreement. After 12 months the license terms will change to a Creative Commons AttributionNonCommercial-Share Alike 4.0 Unported License. 